\title{
CARACTERIZACION MORFOLOGICA DE FRUTOS DE Oenocarpus bataua C. Martius "ungurahui"
}

\author{
Agustín GONZÁLES CORAL ${ }^{1 *}$, Kember MEJÍA CARHUANCA $^{1}$, Guiseppe TORRES REYNA ${ }^{1}$ \\ 1 Instituto de Investigaciones de la Amazonia Peruana. Av. Abelardo Quiñones km 2.5, Iquitos, Perú. *E-mail: \\ agonzales@iiap.org.pe
}

\section{RESUMEN}

Se realizaron mediciones biométricas en muestras de 125 a 150 frutos de 12 procedencias de Oenocarpus bataua "ungurahui". Se realizó el análisis de varianza de las principales características del fruto con $95 \%$ de confianza y el análisis de medias para las variables peso de fruto y peso de pulpa. Las procedencias mostraron diferencias significativas para todas las características. Las medias de longitud del fruto variaron entre 2,76 a $3,93 \mathrm{~cm}$, diámetro de fruto entre 2,14 a 2,55 cm, peso de fruto entre 8,40 a 15,37 g, peso del epicarpio entre 1,21 a 2,78 g, peso del endocarpo entre 5,79 a 10,18 g, longitud del endocarpo entre 2,44 a 3,54 cm, diámetro del endocarpo entre $1,74 \mathrm{a} 2,28 \mathrm{~cm}$, peso mesocarpo entre 1,33 a 2,71 $\mathrm{g}$ y rendimiento de pulpa entre 21,32 a 25,21\%. El análisis del peso del fruto reportó como las mejores medias a P7 (Agua Blanca 33), P3 (Nueva York1) y P9 (Nueva York), con 15,37g, 14,86 g y 14,46 g respectivamente. Mientras que para el peso de pulpa reporto como mejores a P3, P7 y P9, con 2,71,2,42 y 2,39g respectivamente.

PALABRAS CLAVE: Theobroma subincanun, macambillo, caracterización, FINCyT, IIAP, Amazonia peruana.

\section{MORPHOLOGICAL CHARACTERIZACION OF FRUIT OF Oenocarpus bataua C. Martius "ungurahui"}

\section{ABSTRACT}

Overview biometric measurements were made on samples from 125 to 150 fruits of 12 provenances of Oenocarpus bataua "ungurahui". The analysis of variance of the main characteristics of the fruit of the analysis of means for the variables fruit weight and weight of pulp with $95 \%$ confidence. The provenances showed significant differences for all traits. The average fruit length of ranged from 2.76 to $3.93 \mathrm{~cm}$, diameter of fruit 2.14 to $2.55 \mathrm{~cm}$, weight fruit 8.40 to $15.37 \mathrm{~g}$, weight epicarp 1.21 to $2.78 \mathrm{~g}$, weight endocarp 5.79 to $10.18 \mathrm{~g}$, length endocarp 2.44 to $3.54 \mathrm{~cm}$, diameter endocarp 1.74 to $2.28 \mathrm{~cm}$, weight 1.33 to $2.71 \mathrm{~g}$ mesocarp, 21.32 to $25.21 \%$ pulp yield. Analysis of fruit weight averages reported as the best (33 white water), P3 (new York1) P7 and P9 (New York) with $15.37 \mathrm{~g}, 14.86 \mathrm{~g}$ and $14.46 \mathrm{~g}$ respectively. While for weight of P3, P7 and P9 pulp with 2.71,2.42 and $2.39 \mathrm{~g}$ respectively

KEYWORDS: Oenocarpus bataua, ungurahui, characterization, FINCyT, Peruvian Amazon, IIAP. 


\section{INTRODUCCIÓN}

La palmera ungurahui Oenocarpus bataua, sin. Jessenia bataua subsp. batana (Brako \& Zarucchi, 1996) (Montúfar \& Pintaud, 2008), ocurre en casi toda la cuenca amazónica, tanto en suelos inundados y encharcados de tierra firme, como en suelos no inundados de tierra firme, siendo vista como una alternativa al aceite de oliva, pues su perfil de ácidos grasos es muy similar (Clement et al., 2005; Trevejo et al., 2003; Briceño \& Navas, 2005). Oenocarpus bataua es una palmera de tronco solitario y recto, que alcanza los 25 a 30 metros de altura. En la Amazonía peruana, los frutos se consumen directamente o en forma de bebida o refresco bastante agradable de sabor a chocolate (Trevejo, 2003). Las tortas y harinas del ungurahui, que en su mayoría contienen fibra, proteína y carbohidratos, muestran cualidades que pueden ser aprovechadas en la industria de insumos e ingredientes alimentarios (Quispe, et al, 2009). El objetivo de este trabajo es presentar avances en la caracterización morfológica de frutos de $O$. bataua C. Martius, en el marco del proyecto caracterización y selección de poblaciones de cinco especies nativas amazónicas con potencial agroindustrial, convenio IIAP-FINCYT.

\section{MATERIALES Y MÉTODOS}

Se realizaron mediciones biométricas de 125 a 150 frutos maduros elegidos al azar de los racimos cosechados por procedencia. Los frutos presentaban características de mesocarpo de color morado y mesocarpo de color crema (tabla 1). Se tomaron mediciones de longitud de fruto, diámetro de fruto, peso de fruto, peso de cáscara, peso de pulpa, largo semilla, diámetro de semilla, peso de una semilla, \% de pulpa, $\%$ de cascara, $\%$ semilla , color de fruto y color de pulpa (Quispe et al, 2009) (Gonzales, 2007). Para el análisis estadístico de los datos del peso de fruto, peso de pulpa, largo de fruto y diámetro de frutos, se utilizó el análisis de varianza con $95 \%$ de confianza. Los datos fueron procesados a través de los Programas Pasw Statistic 18 y MINITAB 16.

\section{RESULTADOS Y DISCUSIONES}

Miranda et al (2008), menciona que cada fruto maduro de $O$. bataua alcanza los $4 \mathrm{~cm}$. de largo y entre 1,7 a 2,2 cm de ancho, la parte carnosa del fruto es oleaginosa, de color blanco, rojizo o violeta claro. Asimismo, Díaz \& Ávila (2002), describen al fruto como oblongo o elipsoide de 2,5 a 3,5 cm de largo y 2,0 a $2,5 \mathrm{~cm}$ de diámetro, el mesocarpio carnoso, oleaginoso, de aproximadamente $0,5-1,5 \mathrm{~mm}$ de espesor y de color entre blanco y violeta con elevado contenido de aceite. Los resultados reportaron medias de longitud de fruto de 2,76 a $3,93 \mathrm{~cm}$, diámetro de fruto de 2,14 a $2,55 \mathrm{~cm}$, peso total de fruto de 8,40 a 15,37 g, peso de cáscara de 1,21 a 2,78 g, peso de semilla de 5,79 a $10,18 \mathrm{~g}$, peso de pulpa de 1,33 a $2,71 \mathrm{~g}$, que representa porcentajes de 21,32 a $25,21 \%$ (Tabla 2). La semilla de $O$. bataua en promedio representa el 55\% del peso total del fruto, mientras que la cáscara y la pulpa reportan valores similares que en promedio alcanzan el 23,9 y $23,4 \%$ del peso total del fruto respectivamente (Tabla 3 ). Las medias del porcentaje de pulpa obtenidas son superiores a las encontradas por Trevejo et al, (1991) y Flores (1997) (Tabla 4). Según el análisis de varianza de 12 procedencias, todas las características mostraron diferencias significativas (Tabla 5)

\section{Análisis de medias de peso de fruto y peso de pulpa.}

El análisis de medias de la característica peso del fruto, nos muestra un límite permisible máximo de $12,72 \mathrm{~g}$ y un mínimo permisible de $11,82 \mathrm{~g}$, con una media general central de 12,27 g. Por encima del límite máximo permisible se proyectan en orden de importancia las procedencias P7 (Agua Blanca 33), P3 (Nueva York1) y P9 (Nueva York) con 15,37, 14,86 y $14,46 \mathrm{~g}$ respectivamente (diagrama 1). Para la característica peso de pulpa, el análisis nos muestra un límite permisible máximo de $2,19 \mathrm{~g}$ y un mínimo permisible de 1,73 g, con una media general central de 1,96 g. Por encima del límite máximo permisible se proyectan en orden de importancia las procedencias P3, P7 y P9 con 2,71, 2,42 y 2,39 g respectivamente (Gráfico 1 y 2). 
Tabla 1. Datos de origen de 12 accesiones de Oenocarpus bataua $C$. Martius ungurahui, procedentes de la amazonía Peruana.

\begin{tabular}{lllccc}
\hline \multirow{2}{*}{ Procedencia } & \multirow{2}{*}{ Cuenca } & \multicolumn{2}{c}{ Coordenadas } & Altitud \\
\cline { 4 - 5 } & & UTM & 18M & (m.s.n.m.) \\
\hline P1 & AGUA BLANCA PARC. 33 & RíO ITAYA & 9534528 & 0658673 & 109 \\
\hline P2 & ALLPAHUAYO 1 & CIN & 9561337 & 0675512 & 116 \\
P3 & NUEVA YORK 1 & Rí́ MOMÓN & 9610650 & 0679713 & 93 \\
\hline P4 & AGUA BLANCA PARC. 38 & RíO ITAYA & 9534672 & 0658526 & 110 \\
\hline P5 & ALLPAHUAYO 2 & CIN & 9561892 & 0674408 & 125 \\
\hline P6 & SAN JUAN UNGURAHUAL1 & Rí́ NANAY & 9569099 & 0631710 & 95 \\
\hline P7 & AGUA BLANCA PARC. 36 & RíO ITAYA & 9533850 & 0660714 & 112 \\
\hline P8 & ALLPAHUAYO 6 & CIN & 9562497 & 0675567 & 125 \\
\hline P9 & NUEVA YORK 2 & RíO MOMÓN & 9612470 & 0680128 & 93 \\
\hline P10 & SAN JUAN UNGURAHUAL 2 & RÍO NANAY & 9569099 & 0631710 & 97 \\
\hline P11 & ALLPAHUAYO 3 & CIN & 9560403 & 0674412 & 129 \\
\hline P12 & ALLPAHUAYO 4 & CIN & 9562633 & 0674925 & 125 \\
\hline
\end{tabular}

Tabla 2. Media de características morfológicas de frutos de 12 procedencias Oenocarpus bataua C. Martius

\begin{tabular}{ccccccccc}
\hline & $\begin{array}{c}\text { Largo } \\
\text { fruto } \\
(\mathbf{c m})\end{array}$ & $\begin{array}{c}\text { Diámetro } \\
\text { fruto } \\
(\mathbf{c m})\end{array}$ & $\begin{array}{c}\text { Peso } \\
\text { fruto } \\
(\mathbf{g})\end{array}$ & $\begin{array}{c}\text { Peso } \\
\text { cáscara } \\
\mathbf{( g )}\end{array}$ & $\begin{array}{c}\text { Peso } \\
\text { semilla } \\
\mathbf{( g )}\end{array}$ & $\begin{array}{c}\text { Peso } \\
\text { pulpa } \\
\mathbf{( g )}\end{array}$ & $\begin{array}{c}\text { Largo } \\
\text { semilla } \\
(\mathbf{c m})\end{array}$ & $\begin{array}{c}\text { Diámetro } \\
\text { semilla } \\
\text { (cm) }\end{array}$ \\
\hline P1 & 3,24 & 2,40 & 12,70 & 2,48 & 8,49 & 1,79 & 2,94 & 2,28 \\
P2 & 2,84 & 2,44 & 11,32 & 1,64 & 7,85 & 1,83 & 2,60 & 2,08 \\
P3 & 3,58 & 2,46 & 14,86 & 2,39 & 9,75 & 2,71 & 3,29 & 2,08 \\
P4 & 3,49 & 2,38 & 13,21 & 2,42 & 8,87 & 1,92 & 3,19 & 2,09 \\
P5 & 3,06 & 2,51 & 12,36 & 1,84 & 8,44 & 2,08 & 2,79 & 2,06 \\
P6 & 2,94 & 2,17 & 9,67 & 1,70 & 6,51 & 1,46 & 2,68 & 1,79 \\
P7 & 3,66 & 2,48 & 15,37 & 2,78 & 10,18 & 2,42 & 3,36 & 2,12 \\
P8 & 2,82 & 2,14 & 8,40 & 1,21 & 5,79 & 1,40 & 2,51 & 1,74 \\
P9 & 3,51 & 2,44 & 14,46 & 2,41 & 9,65 & 2,39 & 3,19 & 2,06 \\
\hline P10 & 2,96 & 2,29 & 9,72 & 1,77 & 6,62 & 1,33 & 2,69 & 1,80 \\
P11 & 2,76 & 2,40 & 10,75 & 1,64 & 7,24 & 1,87 & 2,44 & 1,97 \\
\hline P12 & 3,93 & 2,55 & 13,60 & 1,96 & 9,36 & 2,28 & 3,54 & 2,03 \\
\hline Total & 3,23 & 2,39 & 12,20 & 2,02 & 8,23 & 1,96 & 2,94 & 2,01 \\
\hline
\end{tabular}


Tabla 1. Composición porcentual del fruto de 12 procedencias de 0 , bataua.

\begin{tabular}{lccc}
\hline & $\begin{array}{c}\% \\
\text { cáscara }\end{array}$ & $\begin{array}{c}\% \\
\text { semilla }\end{array}$ & $\begin{array}{c}\% \\
\text { pulpa }\end{array}$ \\
\hline P1 & 26,22 & 54,91 & 21,93 \\
\hline P2 & 23,67 & 54,14 & 25,21 \\
P3 & 22,32 & 56,47 & 23,65 \\
\hline P4 & 25,36 & 55,04 & 22,23 \\
P5 & 22,63 & 55,83 & 24,18 \\
\hline P6 & 24,79 & 55,20 & 22,66 \\
\hline P7 & 25,15 & 54,46 & 23,30 \\
P8 & 22,28 & 56,18 & 24,06 \\
\hline P9 & 24,11 & 54,88 & 23,63 \\
\hline P10 & 25,22 & 55,71 & 21,32 \\
\hline P11 & 22,95 & 55,18 & 24,65 \\
\hline P12 & 22,34 & 56,06 & 24,15 \\
\hline Total & 23,92 & 55,34 & 23,41 \\
\hline
\end{tabular}

Tabla 4. Comparación de la composición porcentual del fruto de 0 . bataua

\begin{tabular}{cccc}
\hline Componente & $\begin{array}{c}\mathbf{1 2} \\
\text { Procedencias }\end{array}$ & $\begin{array}{c}\text { Según Trevejo } \\
\text { et al., 1991 }\end{array}$ & $\begin{array}{c}\text { Según Flores, } \\
\mathbf{1 9 9 7}\end{array}$ \\
\hline Cascara & $22,28-26,22$ & 15,31 & 14,5 \\
Pulpa & $21,32-25,21$ & 15,27 & 19,3 \\
Semilla & $54,14-56,57$ & 69,42 & 66,2 \\
\hline
\end{tabular}


Tabla 5. ANOVA de las principales características de fruto de 12 procedencias de 0. bataua

\begin{tabular}{|c|c|c|c|c|c|c|}
\hline & Factor & $\begin{array}{l}\text { Suma de } \\
\text { cuadrados }\end{array}$ & g.l. & $\begin{array}{l}\text { Media } \\
\text { cuadrática }\end{array}$ & $\mathbf{F}$ & Sig \\
\hline \multirow[t]{3}{*}{ Peso fruto $(\mathrm{g})$} & Procedencia & 1564,158 & 11 & 142,196 & 214,42 & ,00 \\
\hline & Error & 214,198 & 323 &, 663 & 4 & 0 \\
\hline & Total & 1778,357 & 334 & & & \\
\hline \multirow[t]{3}{*}{ Peso pulpa (g) } & Procedencia & 60,617 & 11 & 5,511 & 31,780 &, 00 \\
\hline & Error & 56,009 & 323 & ,173 & & 0 \\
\hline & Total & 116,626 & 334 & & & \\
\hline \multirow[t]{3}{*}{ Largo fruto $(\mathrm{cm})$} & Procedencia & 44,668 & 11 & 4,061 & 376,04 &, 00 \\
\hline & Error & 3,488 & 323 & ,011 & 5 & 0 \\
\hline & Total & 48,156 & 334 & & & \\
\hline \multirow[t]{3}{*}{ Diámetro fruto $(\mathrm{cm})$} & Procedencia & 4,838 & 11 & ,440 & 8,941 &, 00 \\
\hline & Error & 15,888 & 323 &, 049 & & 0 \\
\hline & Total & 20,726 & 334 & & & \\
\hline \multirow[t]{3}{*}{ Peso cáscara (g) } & Procedencia & 66,663 & 11 & 6,060 & 126,36 &, 00 \\
\hline & Error & 15,490 & 323 & ,048 & 8 & 0 \\
\hline & Total & 82,154 & 334 & & & \\
\hline \multirow[t]{3}{*}{ Peso semilla (g) } & Procedencia & 634,574 & 11 & 57,689 & 185,70 & , 00 \\
\hline & Error & 100,340 & 323 &, 311 & 2 & 0 \\
\hline & Total & 734,914 & 334 & & & \\
\hline \multirow[t]{3}{*}{ Largo semilla $(\mathrm{cm})$} & Procedencia & 40,049 & 11 & 3,641 & 357,70 & 00 \\
\hline & Error & 3,288 & 323 &, 010 & 9 & 0 \\
\hline & Total & 43,337 & 334 & & & \\
\hline \multirow[t]{3}{*}{ Diámetro semilla $(\mathrm{cm})$} & Procedencia & 7,644 & 11 & ,695 & 5,725 & 00 \\
\hline & Error & 39,207 & 323 & 121 & & 0 \\
\hline & Total & 46,850 & 334 & & & \\
\hline
\end{tabular}


Análisis de Medias Peso Fruto (g)

Alpha $=0.05$

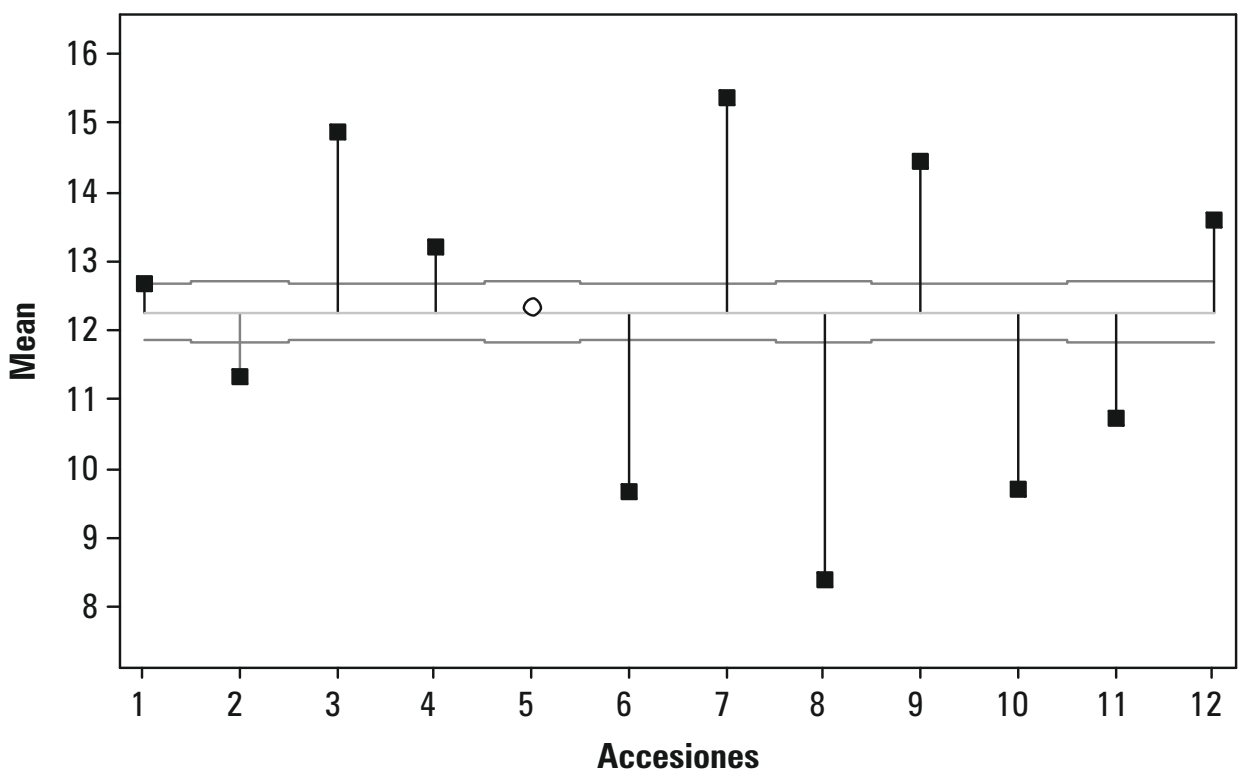

12.720

12.269

11.818

Gráfico 1. Análisis de medias de peso de fruto por procedencias de 0 . bataua

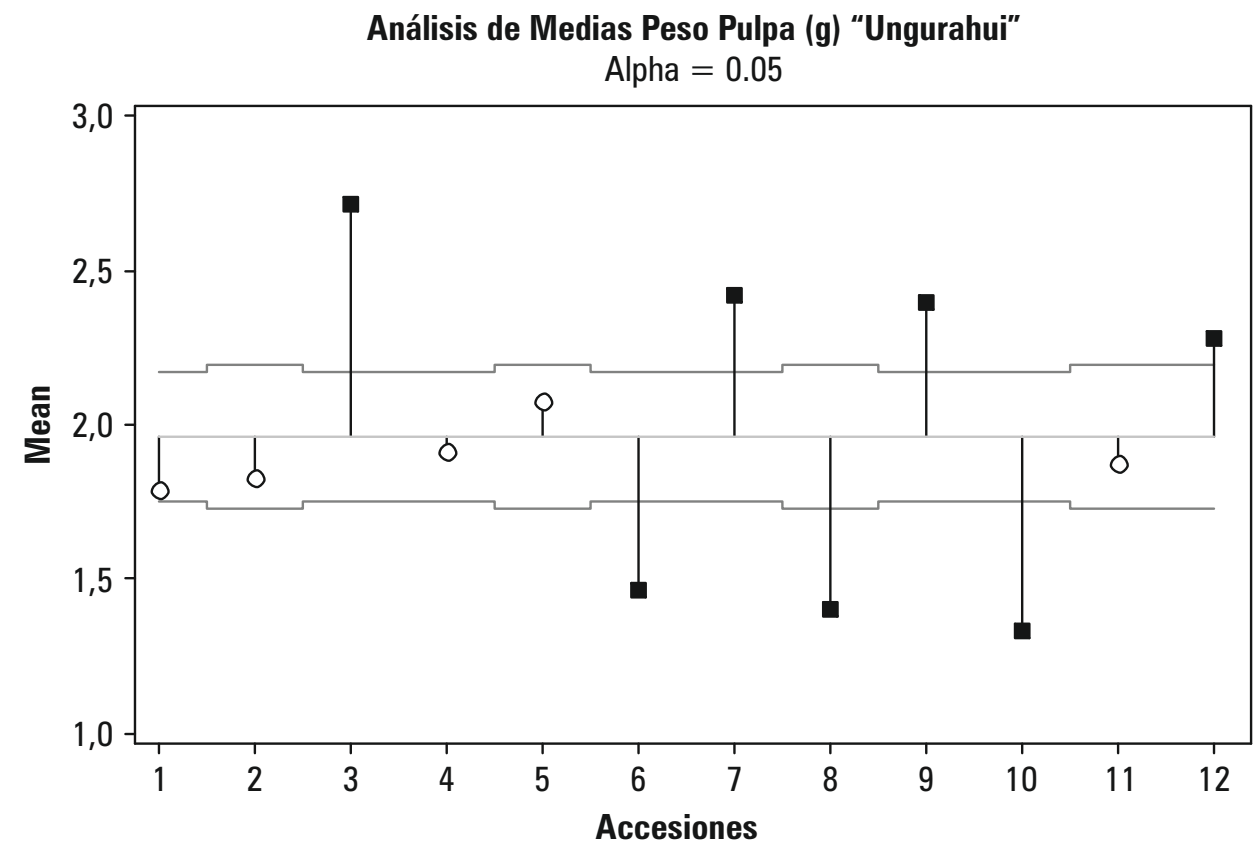

Gráfico 2. Análisis de medias de peso de pulpa por procedencias de 0 . bataua 


\section{CONCLUSIONES}

Todas las características mostraron diferencias significativas entre procedencias al $95 \%$ de confianza. La caracterización muestra procedencias con promedios superiores a los encontrados por otros autores. Esto permitirá en cierto grado tener material de propagación procedente de poblaciones con características deseables sobresalientes. El análisis de medias de peso de fruto y peso de pulpa reportó como las mejores procedencias P7 (Agua Blanca 33), P3 (Nueva York1) y P9 (Nueva York).

\section{BIBLIOGRAFÍA}

Belén, C.; Alviomar, C.; Moreno, Á.; García, D.; Medina, C. 2005. Características físicas y químicas del fruto de la coroba (Jessenia polycarpa Karst): una especie oleífera venezolana. Grasas y Aceites, 56 (4): 317-323.

Brako, L.; Zarucchi, J. L. 1996. Catálogo de las angiospermas y gimnospermas del Perú. Missouri Botanical Garden.

Briceño, M. J. V.; Navas, H. P. V. 2005. Comparación de las características químicas, físicas y perfil de ácidos grasos de los aceites de seje, oliva, maíz y soja. Revista Facultad de Agronomía. Universidad Central de Venezuela (Maracay) 31:109-119.

Clement, C.R.; Lleras Pérez, E.; van Leeuwen, J. 2005. O potencial das palmeiras tropicais no Brasil: acertos e fracassos das últimas décadas. Agrociencias, Montevideu, 9 (1-2): 67-71.

Collazos, M.; Mejía, M. 1988. Fenologia y poscosecha de mil pesos Jessenia bataua (Mart) Burret. Acta Agronómica, 38 (1): 53-63.

Díaz, J. A.; Ávila, L. M. 2002. Sondeo del mercado mundial de Aceite de Seje (Oenocarpus bataua) Instituto de Investigación de Recursos Biológicos Alexander von Humboldt, Bogota, Colombia. $18 \mathrm{pp}$

Flores, P. 1997. Cultivo de frutales nativos Amazónicos. Manual para el extensionista. Tratado de Cooperación Amazónica; Secretaria
Pro-Tempore, Lima Perú; 307 Pág.

Gonzáles, C. A. (2007). Frutales Nativos Amazónicos: Patrimonio Alimenticio de la humanidad. Instituto de Investigaciones de la Amazonía Peruana.

M. C.T, Mambrim; D. Barrera-Arellano. 1997. Grasas y Aceites, 48 (3): 154-158.

Miranda, J.; Montaño, F.; Zenteno, F.; Nina, H. \& J. Mercado, 2008. El Majo (Oenocarpus bataua): una Alternativa de Biocomercio en Bolivia. TRÓPICO - PNBS - FAN. Ediciones TRÓPICO. La Paz, Bolivia.

Montúfar, R., Pintaud J.C. 2008. Estatus taxonómico de Oenocarpus bataua (Euterpeae, Arecaceae) inferido por secuencias del ADN cloroplástico. Las palmeras en América del Sur. Revista Peruana de Biología, 15 (supl. 1): 73-78.

Quispe, J.; Ayala R.; Ingunza, R.; Landeo, P.; Pascual, CH. 2009. Caracterización de aceites, tortas y harinas de frutos de ungurahui (jessenia polycarpa) y aguaje(mauritia flexuosa l.) de la Amazonía Peruana. Revista de la Sociedad Química del Perú, 75 (2): 243-253.

Trevejo, E. 2003. Avances de la investigación en frutos oleaginosos de la Amazonia Peruana. Estudio del aceite de ungurahui (Jessenia bataua). CONCYTEC - UNAP. Noviembre 2003. Pág. 59-71.

Villachica, H. 1996. Frutales y hortalizas promisorios de la Amazonia. Lima- Perú. Tratado de Cooperación Amazónica. 282286pag.

FAO. 2002. Tratado Internacional sobre los recursos fitogenéticos para la alimentación y la agricultura.

Vallejo, R. D. Darío 2002. "Oenocarpus bataua, seje"; Colombia Amazónica, separata especies promisorias 1. Corporación Colombiana para la Amazonia-Araracuara-COA.

Recibido: 15/05/2014

Aceptado para publicación: 10/07/2014 
